\title{
Microkernel Construction
}

\section{Introduction}

SS2012 


\section{Class Goals}

Provide deeper understanding of OS mechanisms

Introduce L4 principles and concepts

Make you become enthusiastic L4 hackers

Propaganda for OS research at TU Dresden 


\section{Administration}

- Thursday, $4^{\text {th }}$ DS, 2 SWS

- Slides: http://www.tudos.org $\rightarrow$ Teaching $\rightarrow$ Microkernel Construction

- Subscribe to our mailing list: http://www.tudos.org/mailman/listinfo/mkc2012

- In winter term:

- Construction of Microkernel-based Systems (2 SWS)

- Various Labs 


\section{"Monolithic" Kernel System Design}

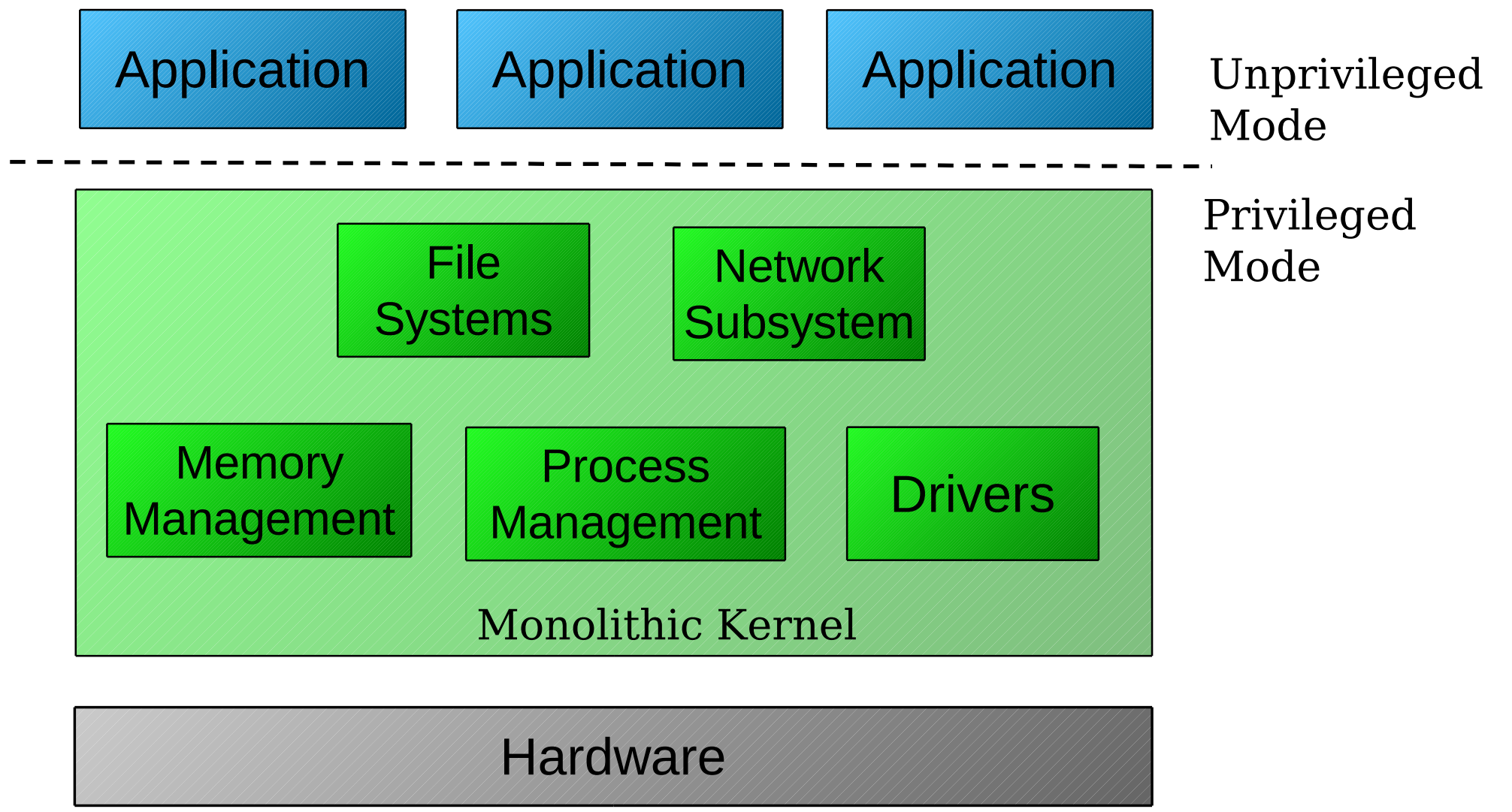

\section{Hardware}




\section{Monolithic Kernel OS (Propaganda)}

- System components run in privileged mode

$\rightarrow$ No protection between system components

- Faulty driver can crash the whole system

- More than 2/3 of today's OS code are drivers

$\rightarrow$ No need for good system design

- Direct access to data structures

- Undocumented and frequently changing interfaces

$\rightarrow$ Big and inflexible

- Difficult to replace system components

Why something different?

- More and more difficult to manage increasing OS complexity 


\section{Microkernel System Design}

\section{Application}

\section{Application}

\section{Application}

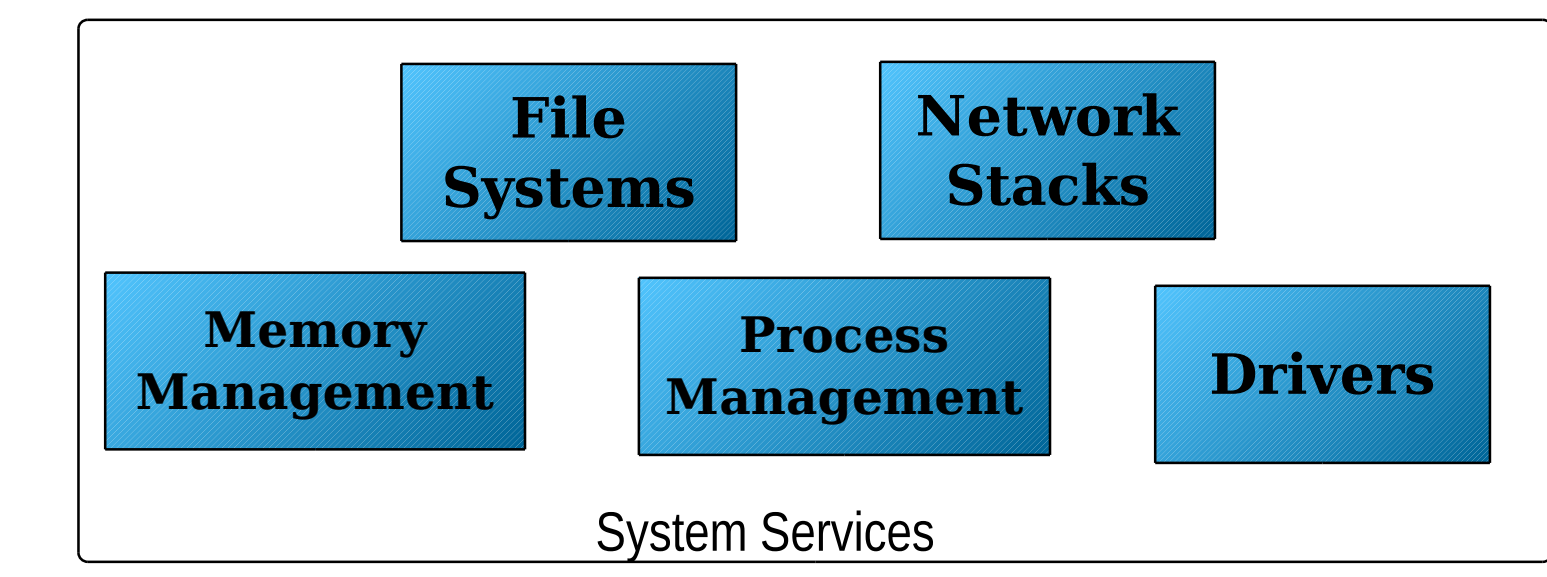

Unprivileged Mode

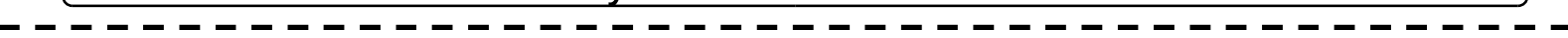

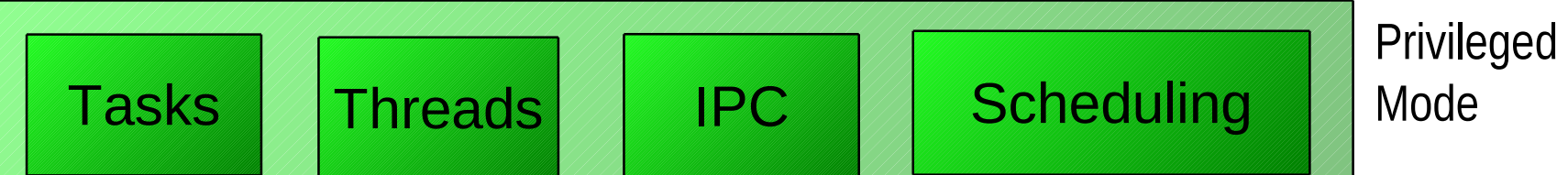

Microkernel

Hardware 


\section{Example - IBM Workplace OS I Mach}

\begin{tabular}{|c|c|c|c|c|}
\hline $\begin{array}{c}\text { OS/2 } \\
\text { Application }\end{array}$ & $\begin{array}{c}\text { DOS } \\
\text { Application }\end{array}$ & $\begin{array}{c}\text { OS/400 } \\
\text { Application }\end{array}$ & $\begin{array}{c}\text { AIX } \\
\text { Application }\end{array}$ & $\begin{array}{l}\text { Windows } \\
\text { Application }\end{array}$ \\
\hline $\begin{array}{c}\text { OS/2 } \\
\text { Personality }\end{array}$ & $\begin{array}{c}\text { DOS } \\
\text { Personality }\end{array}$ & $\begin{array}{l}\text { OS/400 } \\
\text { Personality }\end{array}$ & AIX Personality & $\begin{array}{l}\text { Windows } \\
\text { Personality }\end{array}$ \\
\hline
\end{tabular}

File Server

Default Pager

\section{Network Service}

Device Support
Security

Bootstrap
Power Management

Name Service

\section{Mach Microkernel}

\section{ARM \\ PowerPC \\ IA32 \\ MIPS \\ Alpha}




\section{Example - QNX / Neutrino}

- Embedded systems

- Message passing system (IPC)

- Network transparency

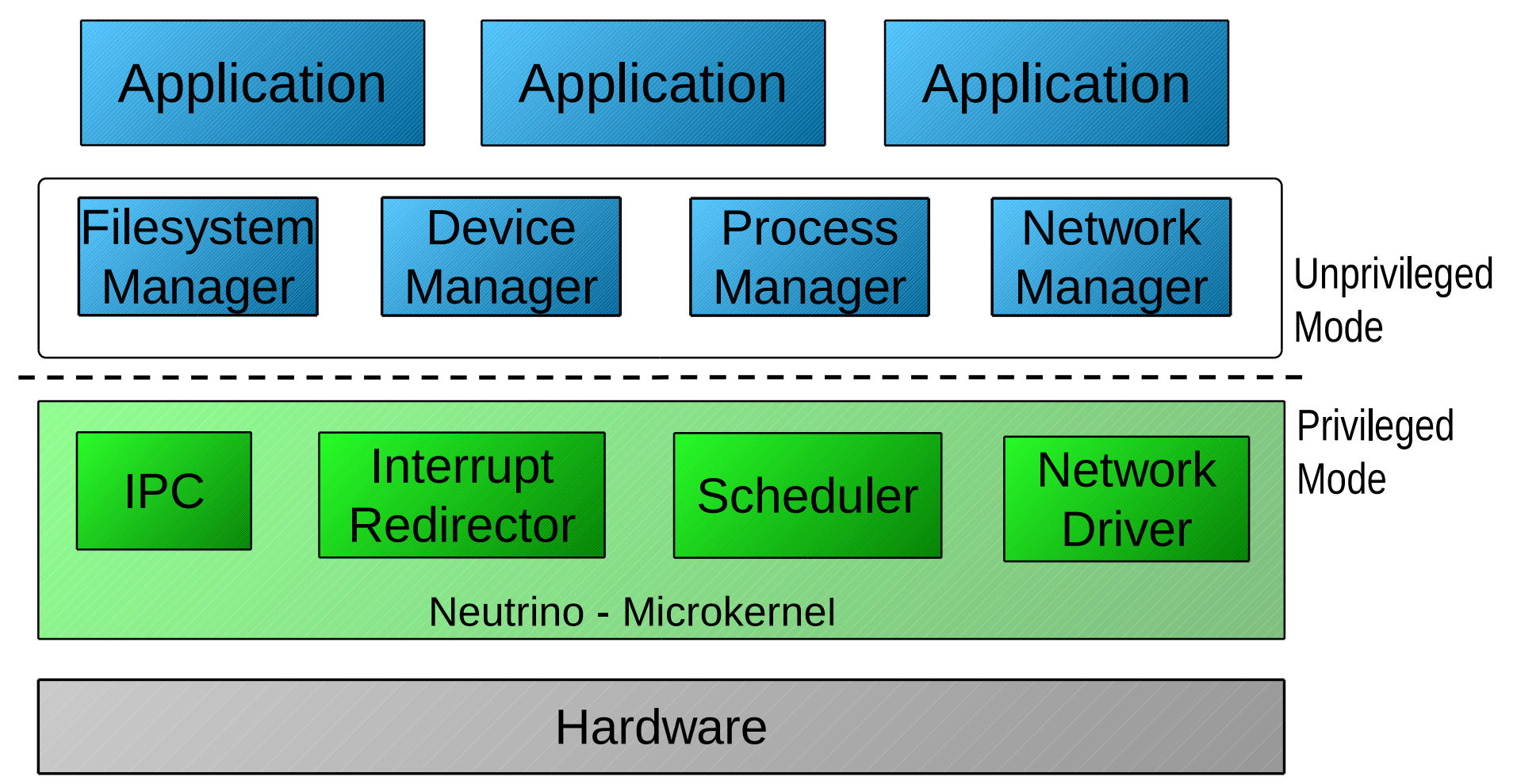

\section{Hardware}




\section{More Interesting}

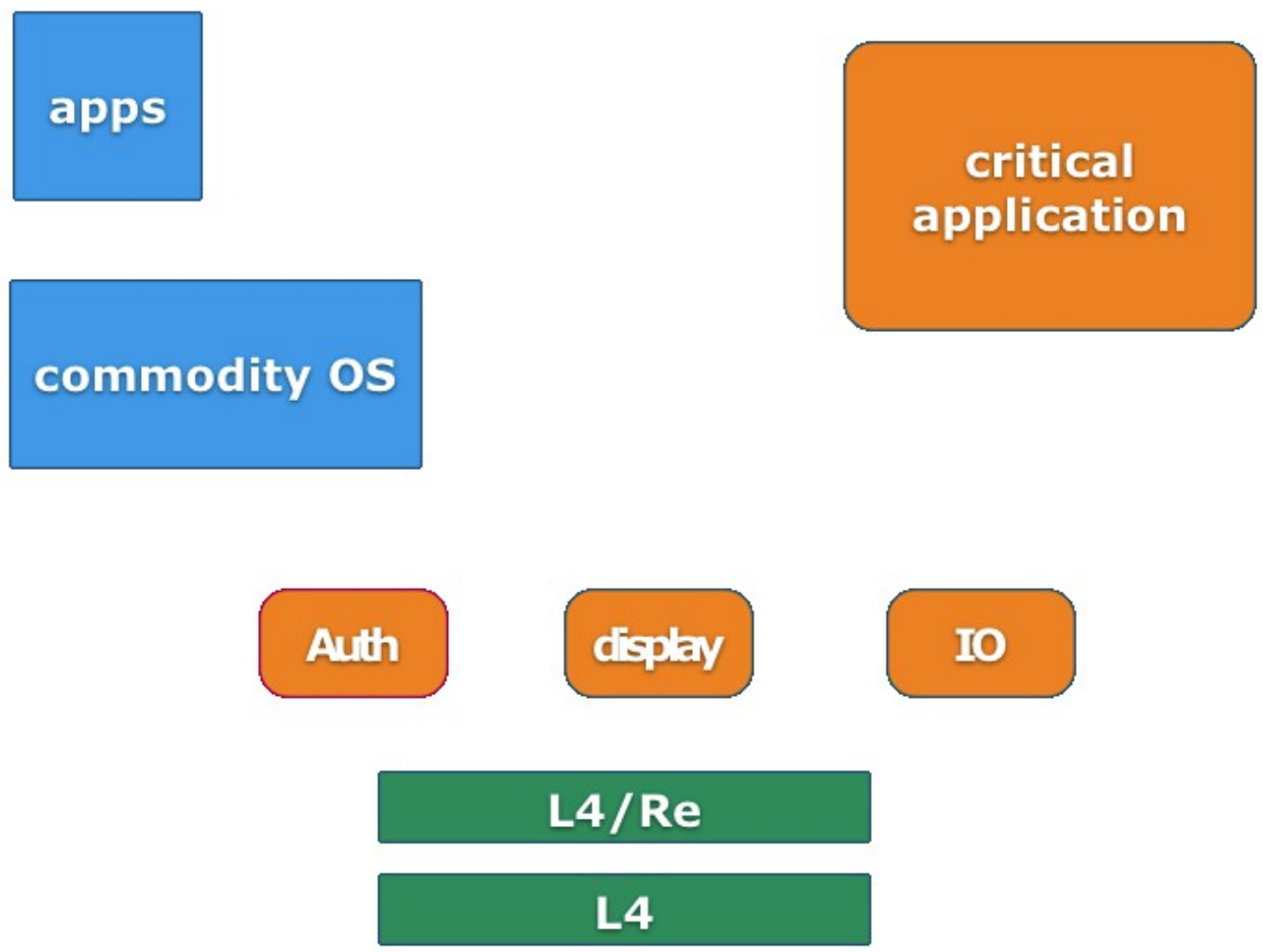

Hermann

Härtig

Torsten

Frenzel

TU Dresden

Operating

Systems Group 


\section{Example: VPN}

Secure Net

Internet

Secure Net

\section{Hermann}

Härtig

Torsten

Frenzel

TU Dresden

Operating

Systems Group
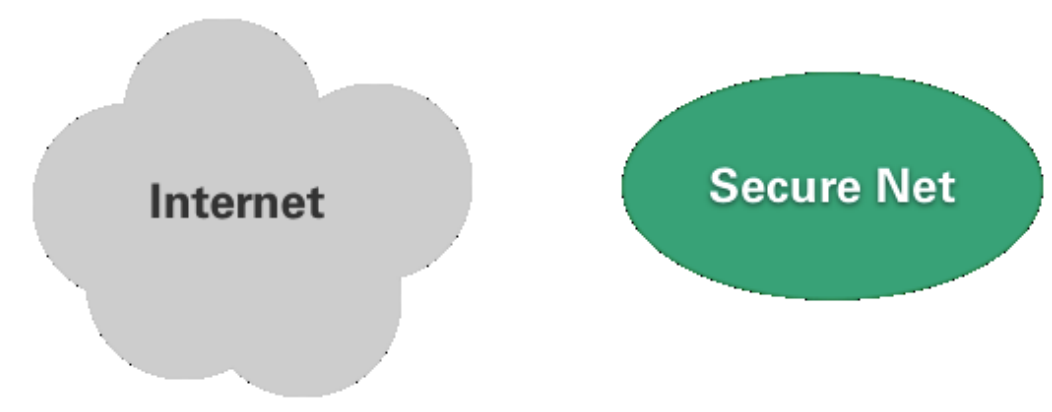


\section{Microkernel}

Construction
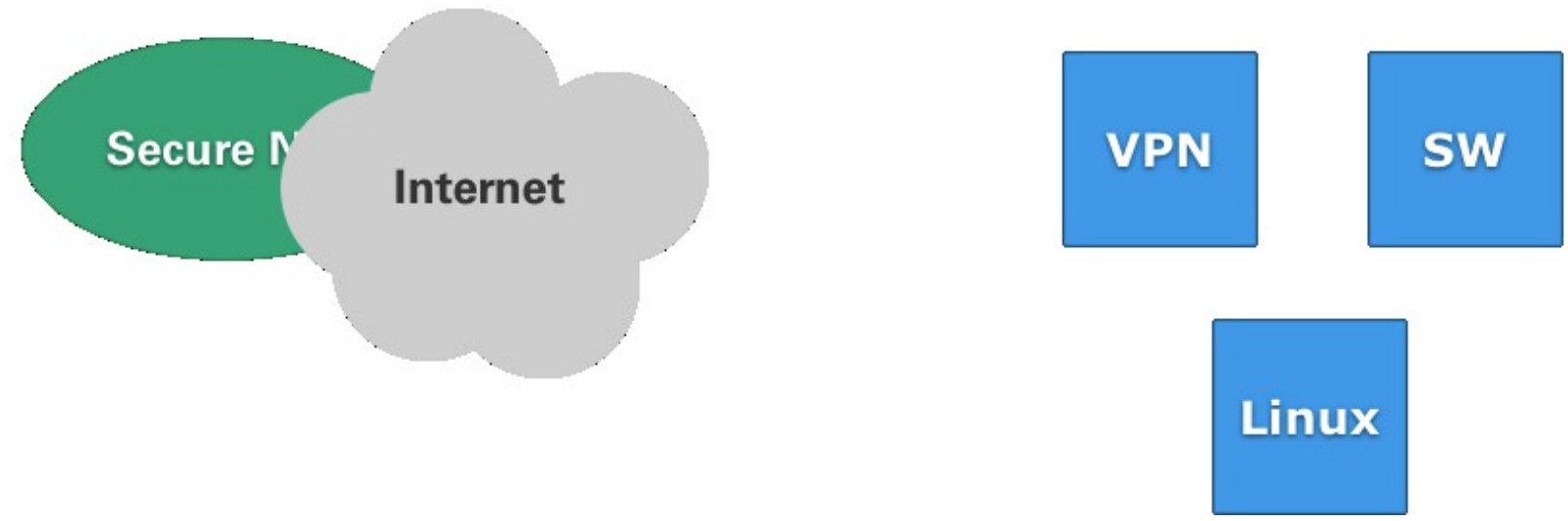

\section{Hermann}

Härtig

Torsten

Frenzel

TU Dresden

Operating

Systems Group 

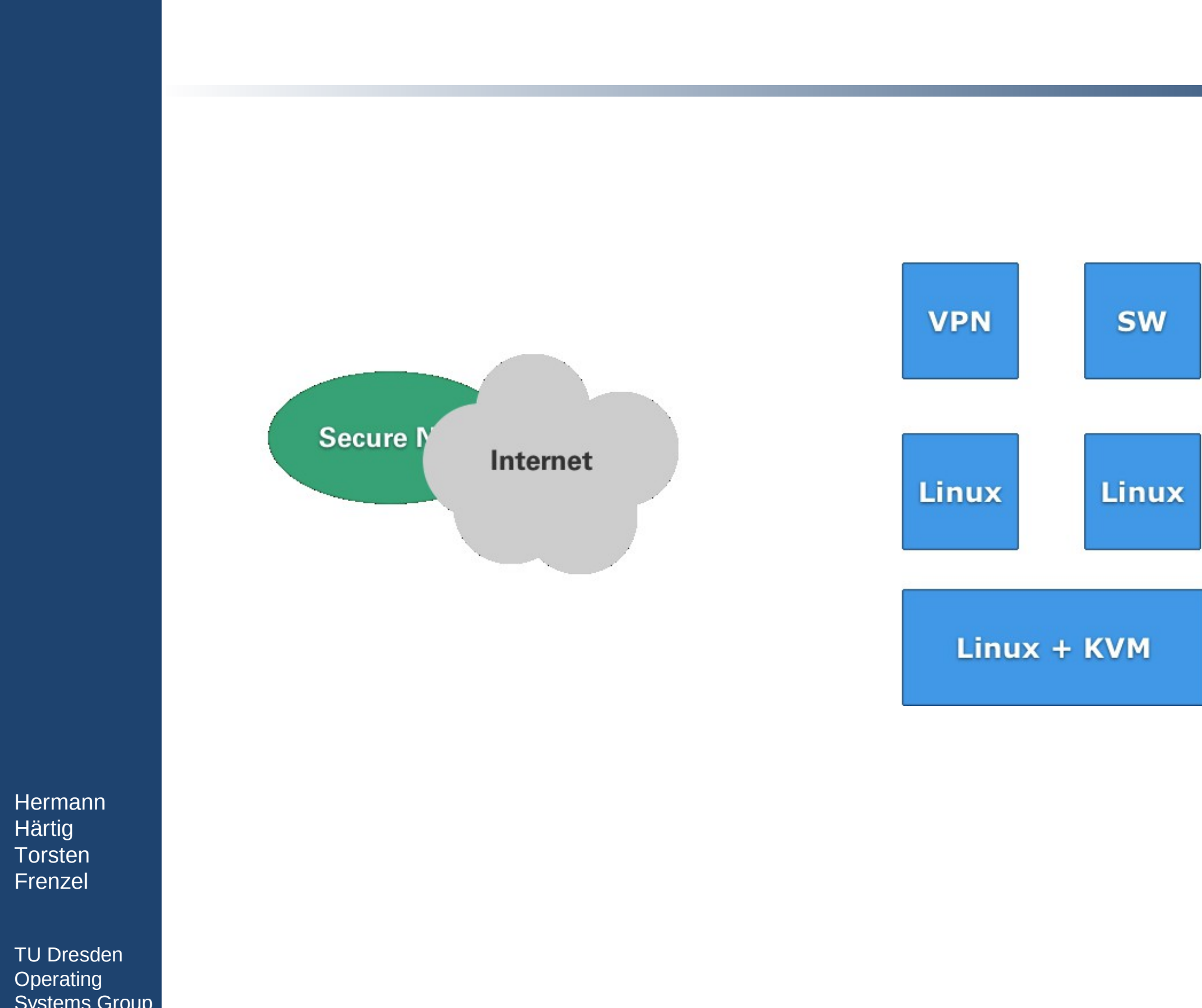

Linux + KVM

Systems Group 

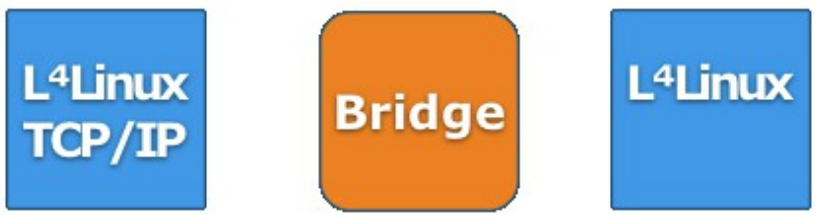

\section{L4}

\section{Hermann}

Härtig

Torsten

Frenzel

TU Dresden

Operating

Systems Group 


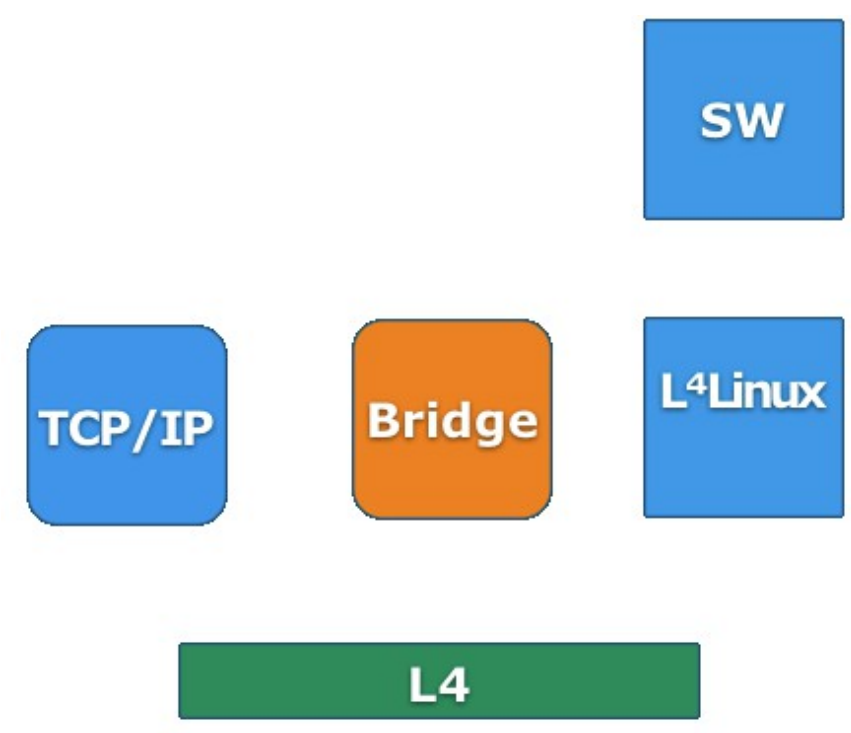

Hermann

Härtig

Torsten

Frenzel

TU Dresden

Operating

Systems Group 


\section{More On Critical Applications}

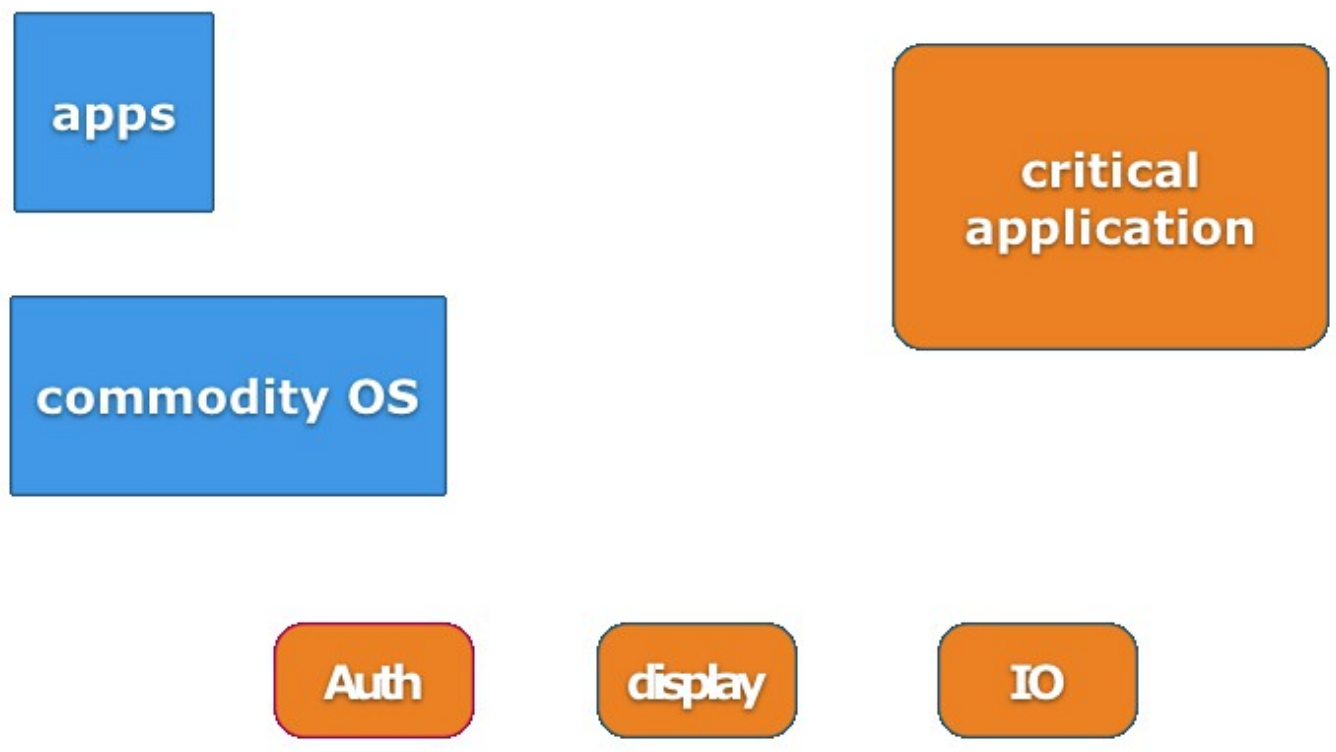

\begin{tabular}{|c|}
\hline $\mathrm{L} 4 / \mathrm{Re}$ \\
\hline $\mathrm{L} 4$ \\
\hline
\end{tabular}

Hermann

Härtig

Torsten

Frenzel

\section{TU Dresden}

Operating 

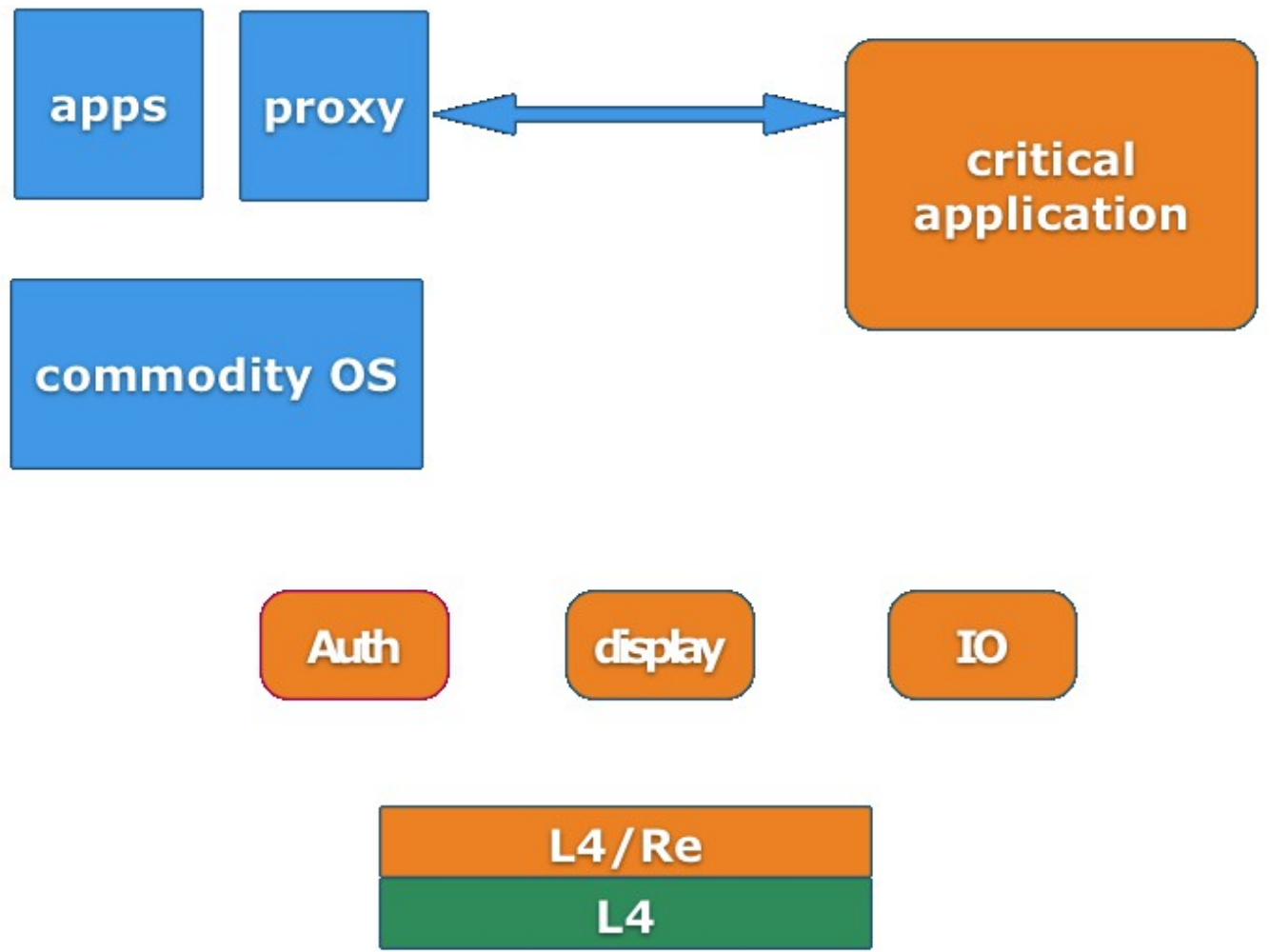

10

Hermann

Härtig

Torsten

Frenzel

TU Dresden

Operating

Systems Group 


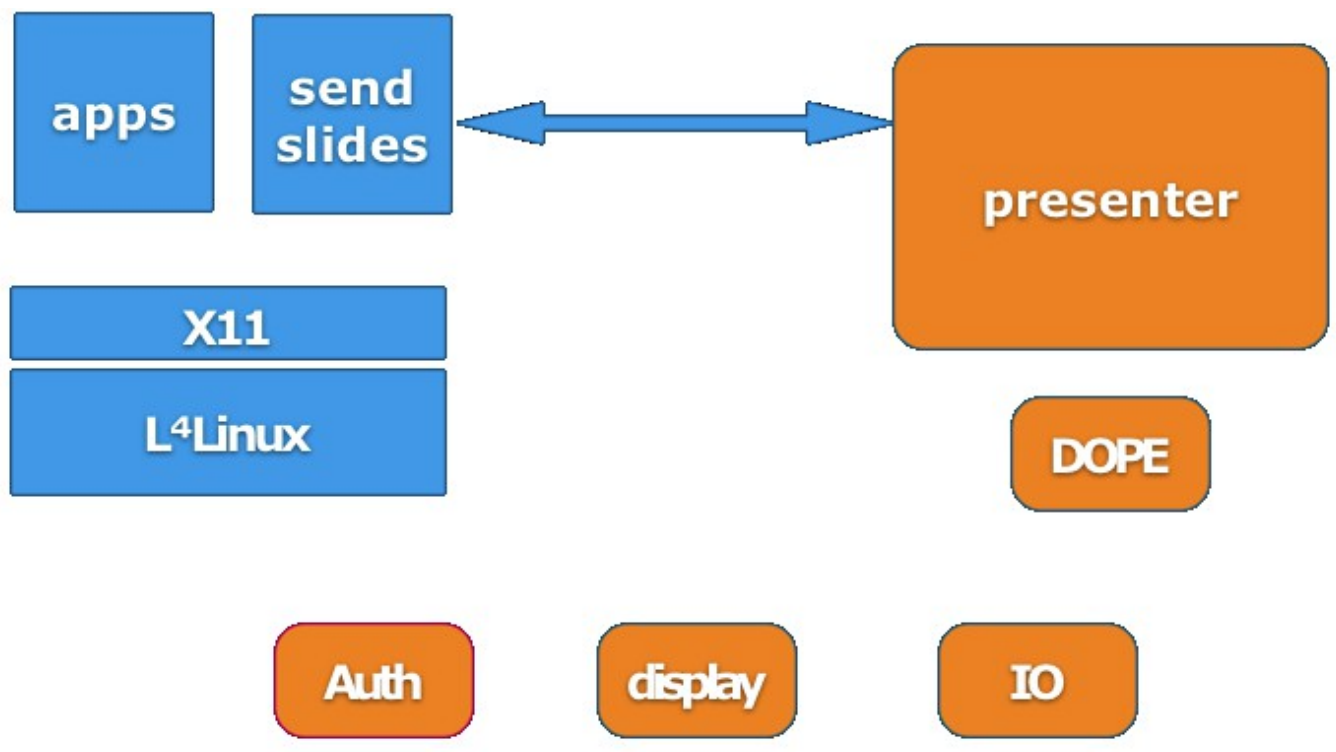

\begin{tabular}{|c|}
\hline $\mathrm{L} 4 / \mathrm{Re}$ \\
\hline $\mathrm{L} 4$ \\
\hline
\end{tabular}

Hermann

Härtig

Torsten

Frenzel

TU Dresden

Operating

Systems Group 


\section{Virtual Private File System}

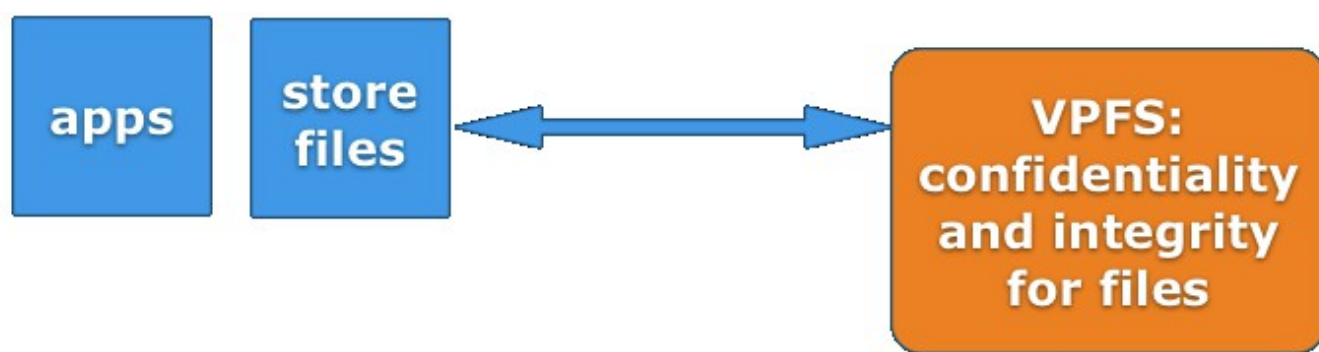

L4Linux

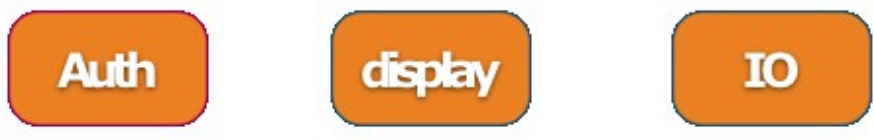

$$
\begin{gathered}
\text { L4/Re } \\
\text { L4 }
\end{gathered}
$$

Frenzel 

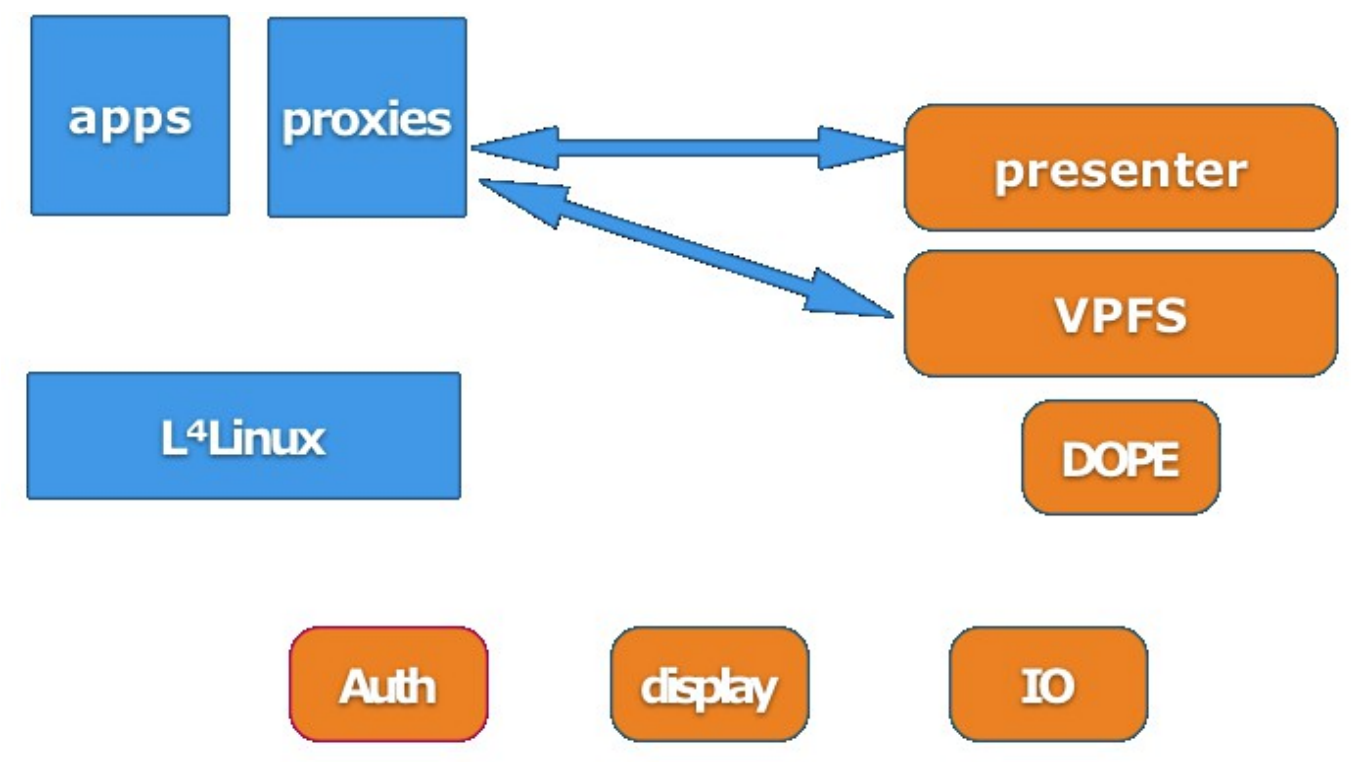

\begin{tabular}{|c|}
\hline $\mathrm{L} 4 / \mathrm{Re}$ \\
\hline $\mathrm{L4}$ \\
\hline
\end{tabular}

Hermann

Härtig

Torsten

Frenzel

TU Dresden

Operating

Systems Group 


\section{Shopping}

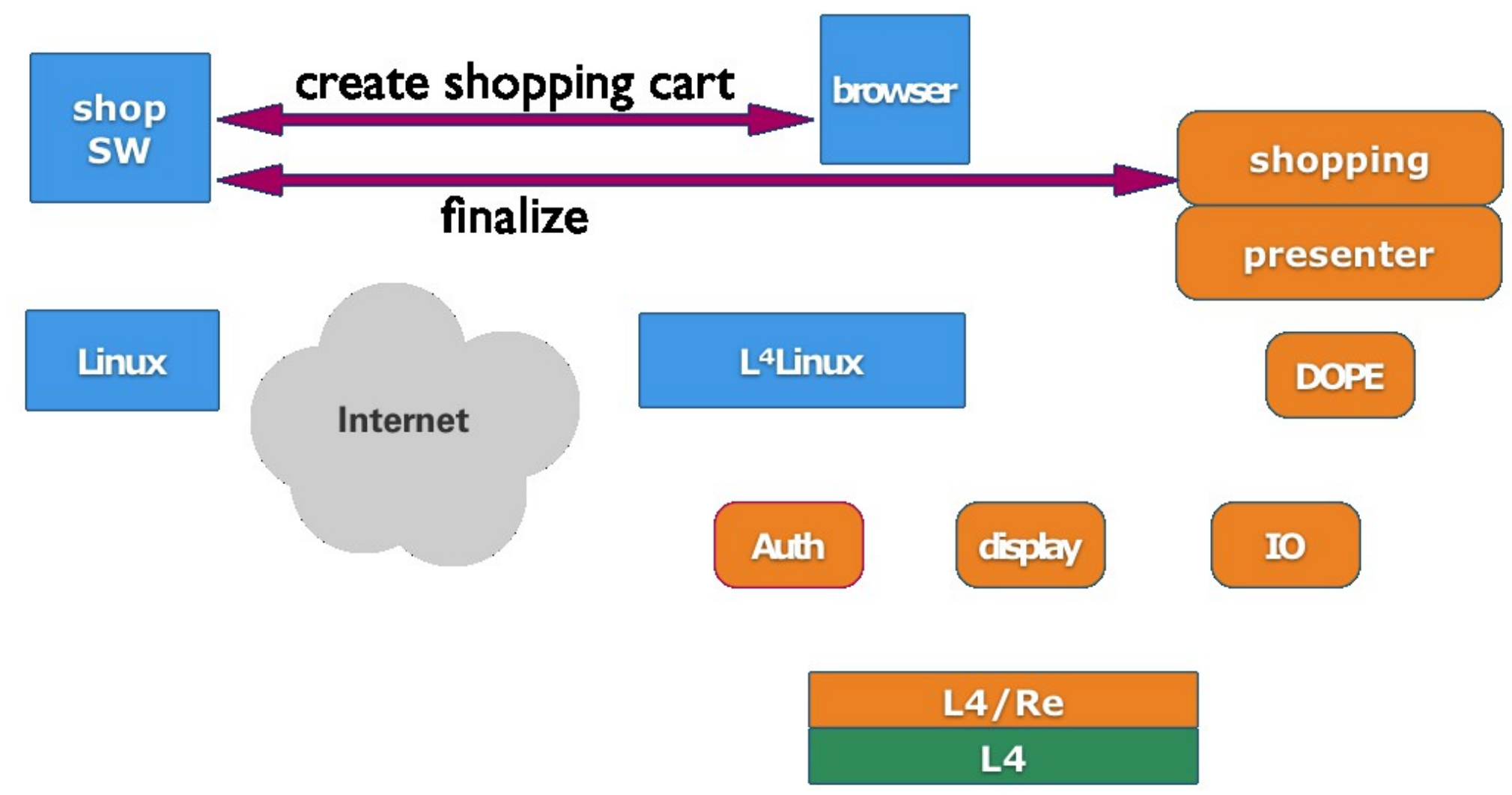




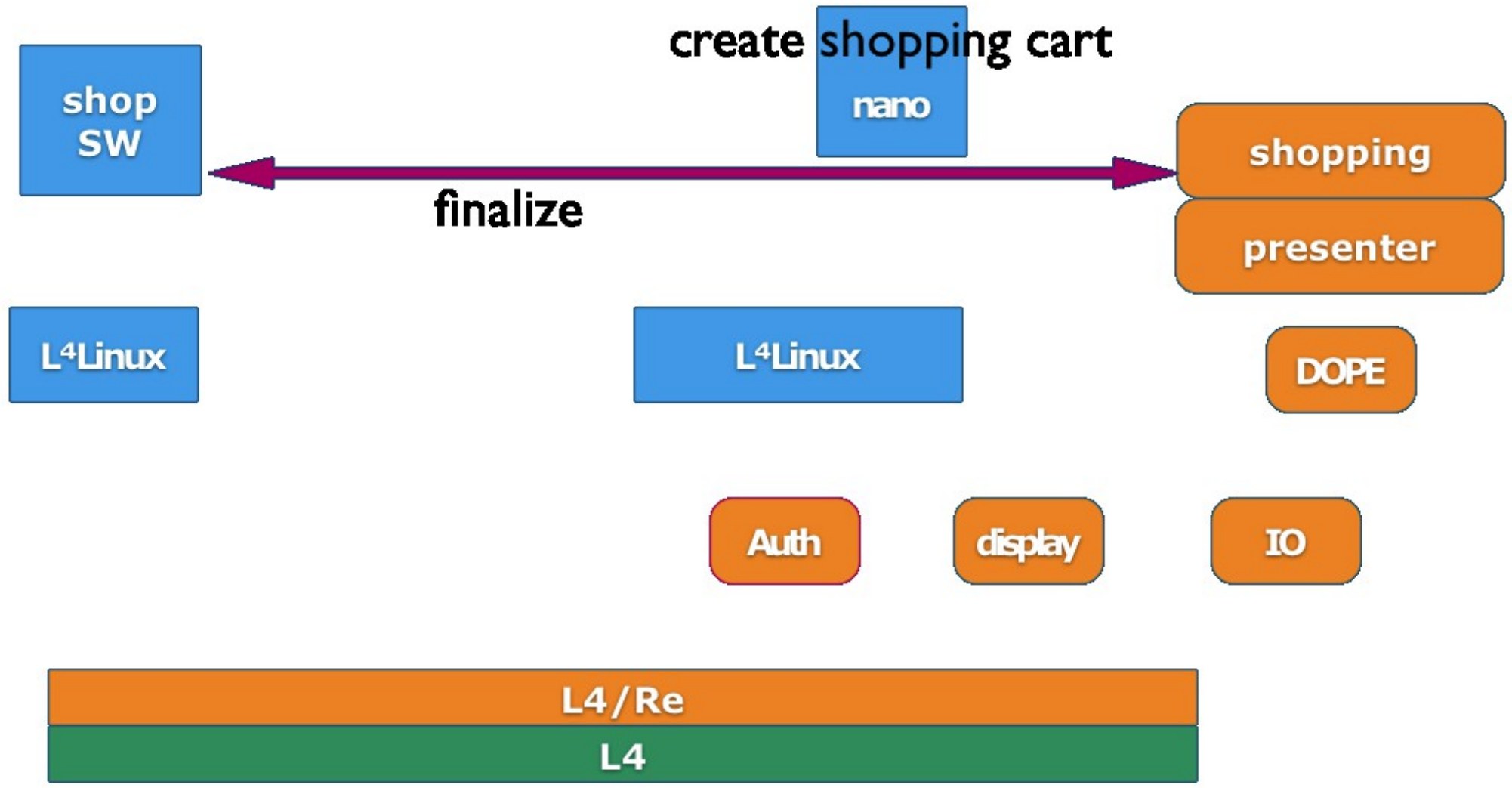




\section{Microkernel OS - The Vision (1)}

- System components run as user-level servers

- Protection and isolation between system components

- More secure / safe systems

- Less error prone

- Small Trusted Computing Base

- „Enforces“ clear system design

- Well defined interfaces to system services

- No dependencies between system services other than explicitly specified through service interfaces

- Small and flexible

- Small OS kernel

- Easier to replace system components 


\section{Visions vs. Reality}

- Flexibility and Customizable

- Monolithic kernels are modular

- Maintainability and complexity

- Monolithic kernel have layered architecture

$\checkmark$ Robustness

- Microkernels are superior due to isolated system components

- Trusted code size (i386)

- Fiasco kernel: about 15.000 loc

- Linux kernel: about 300.000 loc (without drivers)

$x$ Performance

- Application performance degraded

- Communication overhead (see next slides) 


\section{Robustness vs. Performance (1)}

- System calls

- Monolithic kernel: 2 kernel entries/exits

- Microkernel: 4 kernel entries/exits + 2 context switches

Application

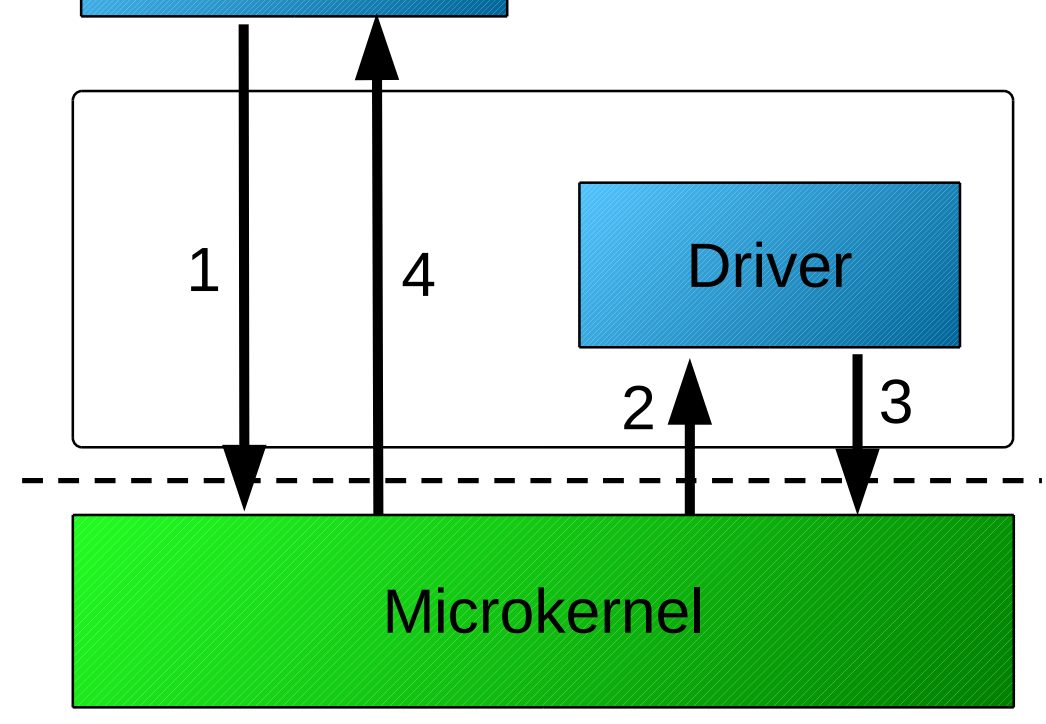

Hardware

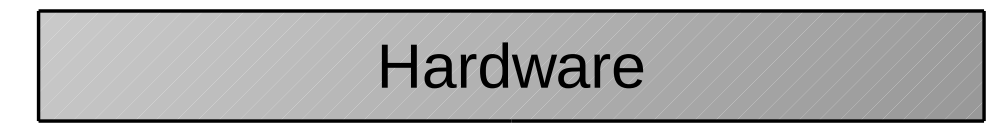

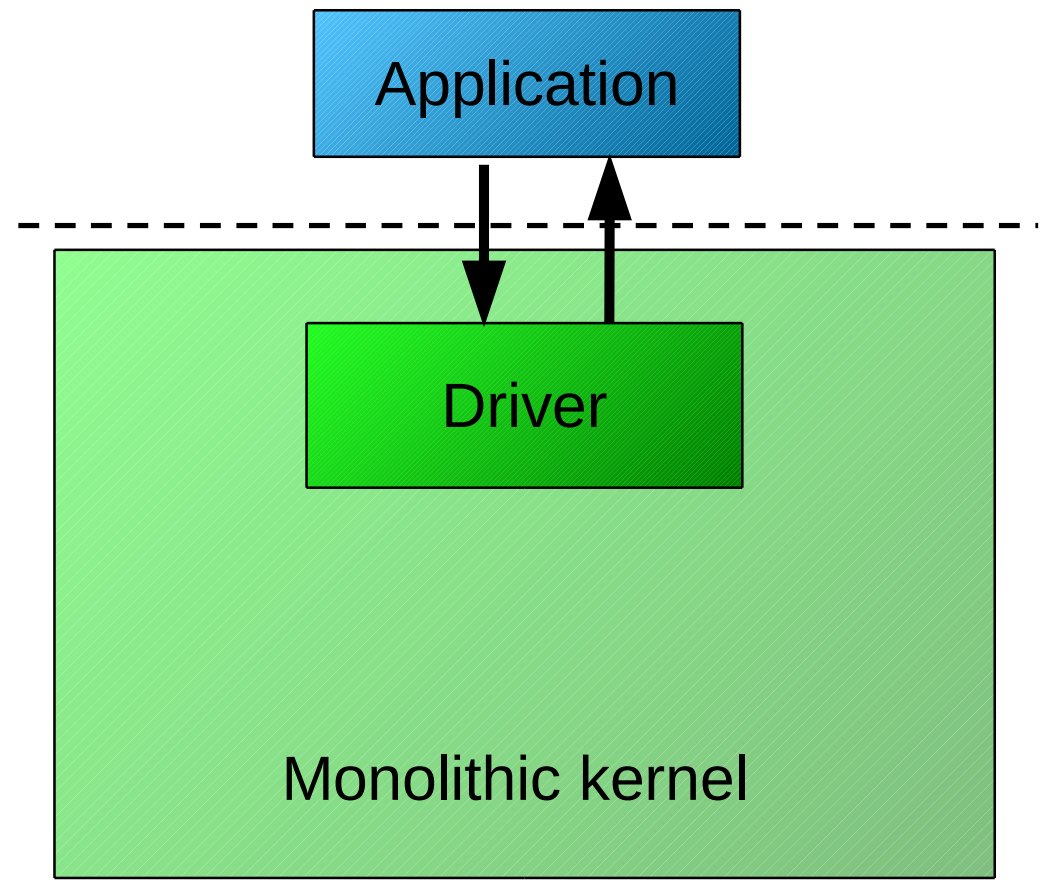

Hardware

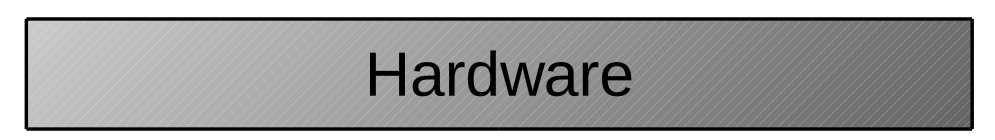




\section{Robustness vs. Performance (2)}

- Calls between system services

- Monolithic kernel: 1 function call

- Microkernel: 4 kernel entries/exits +2 context switches
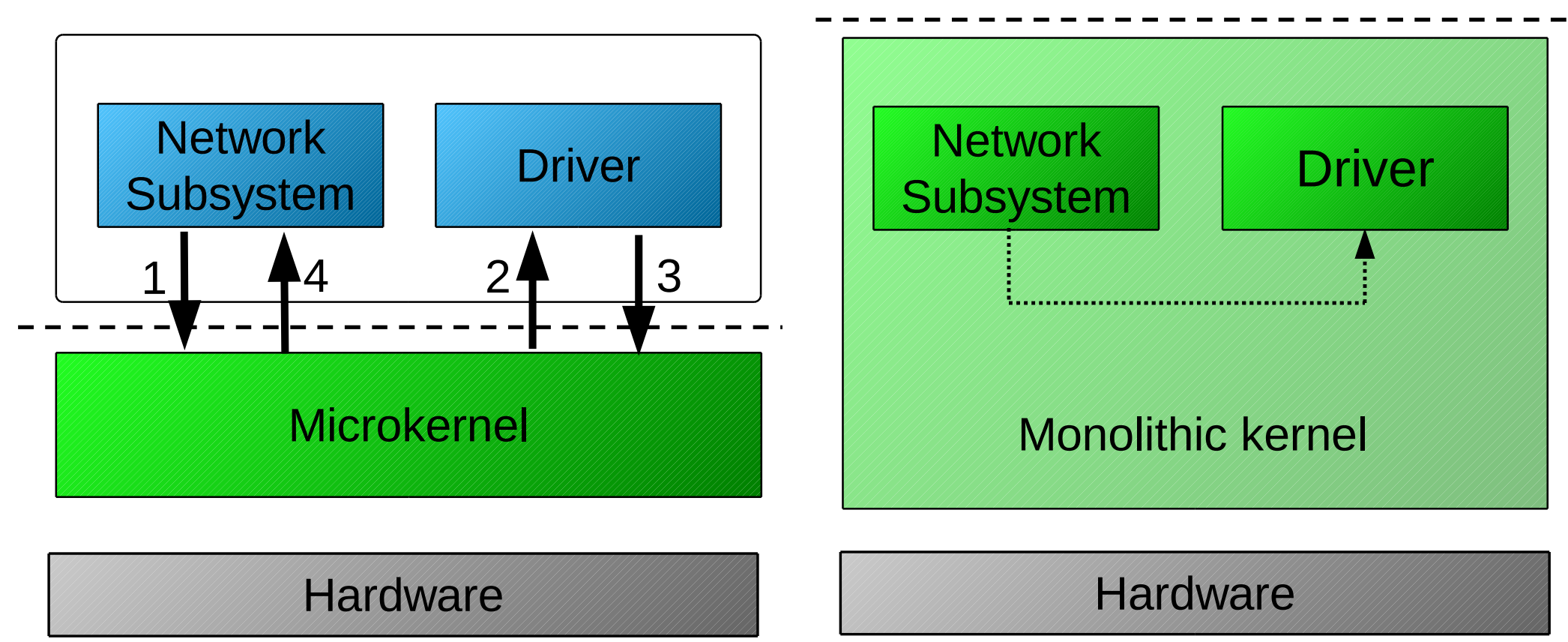


\section{Challenges}

- Build functional powerful and fast microkernels

- Provide abstractions and mechanisms

- Fast communication primitive (IPC)

- Fast context switches and kernel entries/exits

$\rightarrow$ Subject of this lecture

- Build efficient OS services

- Memory Management

- Synchronization

- Device Drivers

- File Systems

- Communication Interfaces

$\rightarrow$ Subject of lecture "Construction of Microkernel-based systems" (in winter term) 


\section{L4 Microkernel Family}

- Originally developed by Jochen Liedtke (GMD / IBM Research)

- Current development:

- Uni Karlsruhe: Pistachio

- UNSW/NICTA/OKLABS: OKL4, SEL4, L4Verified

- TU Dresden: Fiasco.OC, Nova

- Support for hardware architectures:

- X86, ARM, ... 


\section{More Microkernels (Incomplete list)}

- Commercial kernels

- Singularity @ Microsoft Research

- K42@ IBM Research

- velOSity/INTEGRITY @ Green Hills Software

- Chorus/ChorusOS@ Sun Microsystems

- PikeOS@ @YSGO AG

- OKL4

- Research kernels

- EROS/CoyotOS @ John Hopkins University

- Minix@ FU Amsterdam

- Amoeba @ FU Amsterdam

- Pebble @ Bell Labs

- Grasshopper @ University of Sterling

- Flux/Fluke @ University of Utah 


\section{L4 - Concepts}

- Jochen Liedtke: "A microkernel does no real work"

- Kernel provides only inevitable mechanisms

- No policies implemented in the kernel

- Abstractions

- Tasks with address spaces

- Threads executing programs/code

- Mechanisms

- Resource access control

- Scheduling

- Communication (IPC) 


\section{Threads and Tasks}

Task A

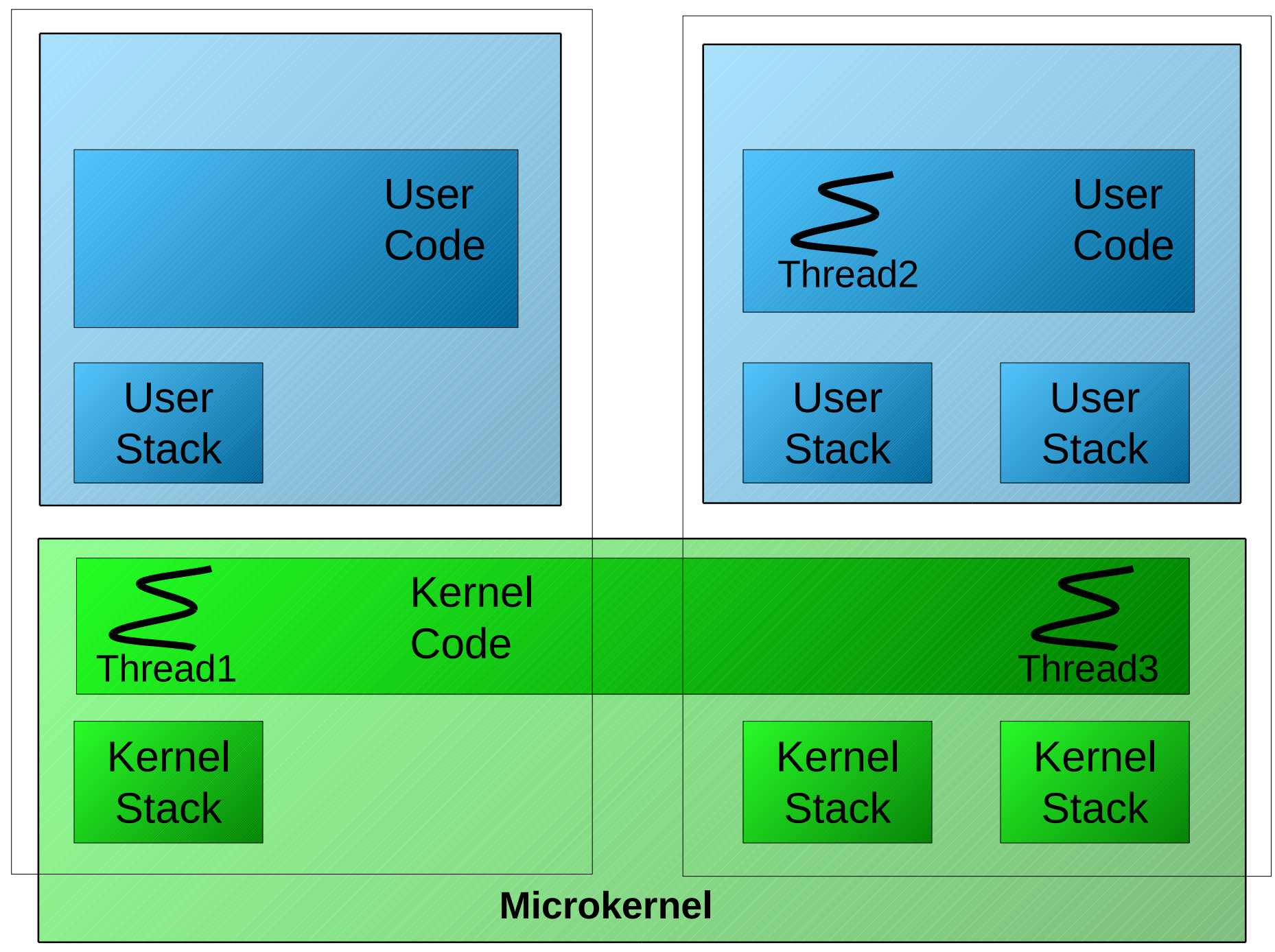

\section{Task B}

Hermann

Härtig

Torsten

Frenzel

TU Dresden

Operating

Systems Group 


\section{Threads (1)}

- Represent unit of execution

- Execute user code (application)

- Execute kernel code (system calls, page faults, interrupts, exceptions)

- Subject to scheduling

- Quasi-parallel execution on one CPU

- Parallel execution on multiple CPUs

- Voluntarily switch to another thread possible

- Preemptive scheduling by the kernel according to certain parameters

- Associated with an address space

- Executes code in one task at one point in time

- Migration allows threads move to another task

- Several threads can execute in one task 


\section{Tasks (1)}

- Represent domain of protection and isolation

- Container for code, data and resources

- Address space: capabilities + memory pages

- Three management operations:

- Map: share page with other address space

- Grant: give page to other address space

- Unmap: revoke previously mapped page
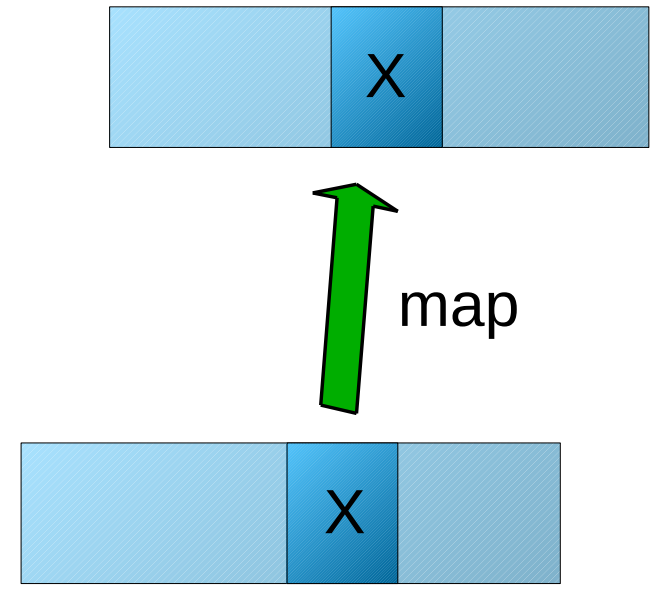

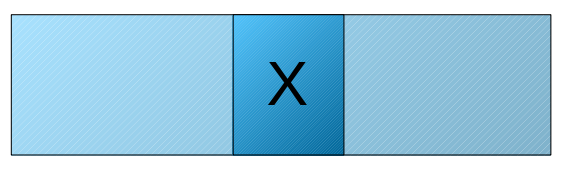

grant

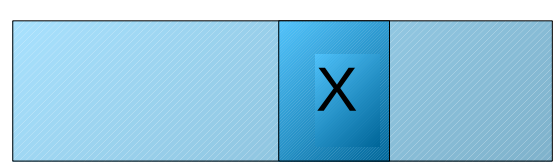

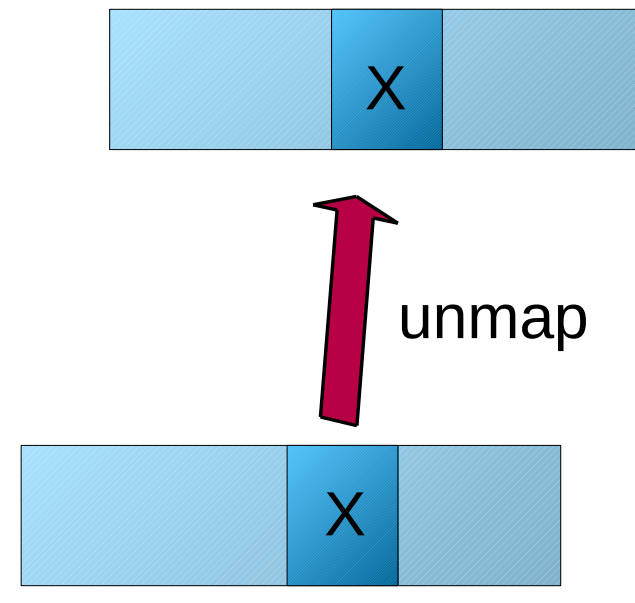




\section{Recursive Address Spaces}

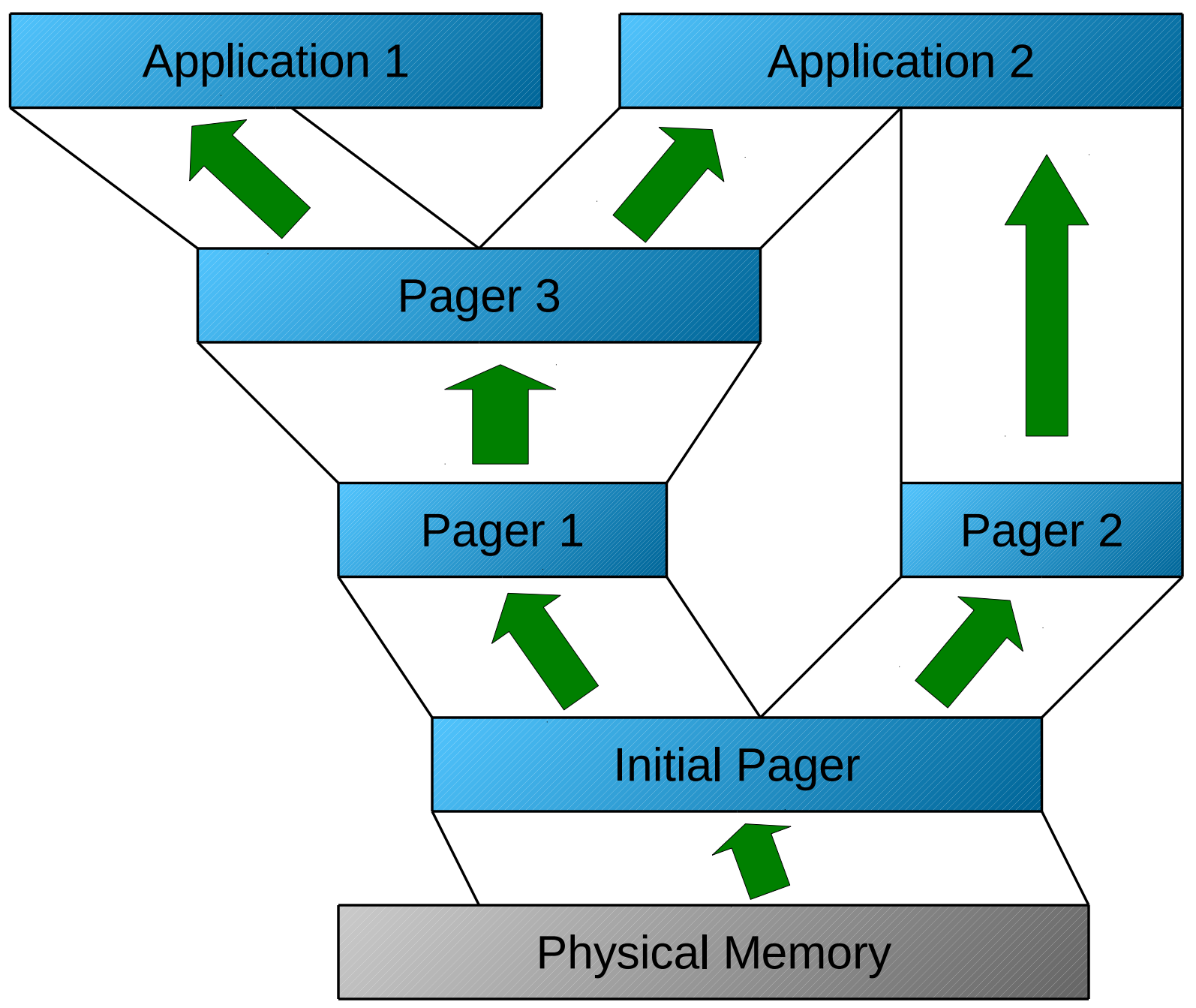

TU Dresden 


\section{Messages: Copy Data}

- Direct and indirect data copy

- UTCB message (special area)

- Special case: register-only message

- Pagefaults during user-level memory access possible

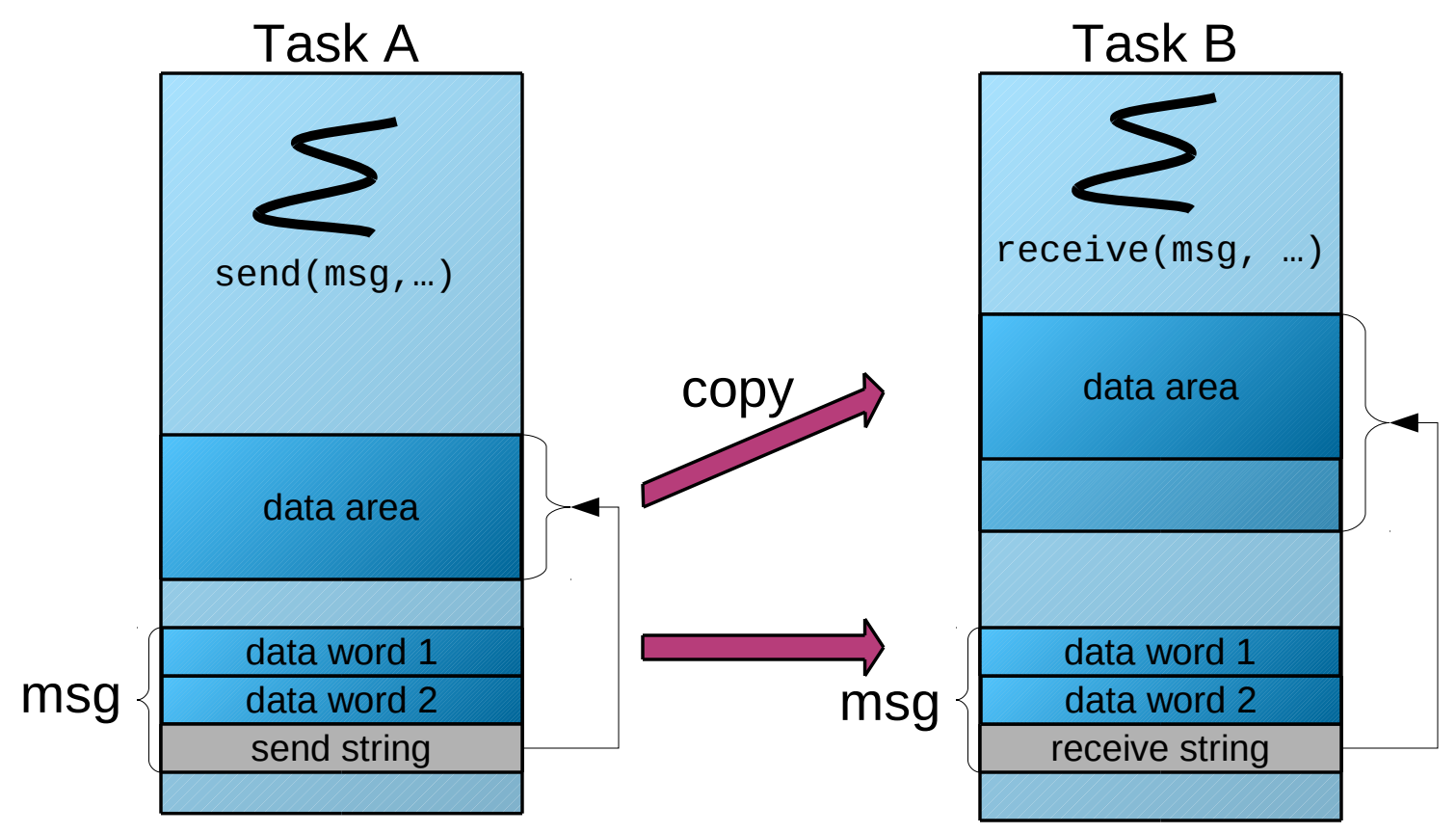




\section{Message: Map References}

- Used to transfer memory pages and capabilities

- Kernel manipulates page tables

- Used to implement the map/grant operations

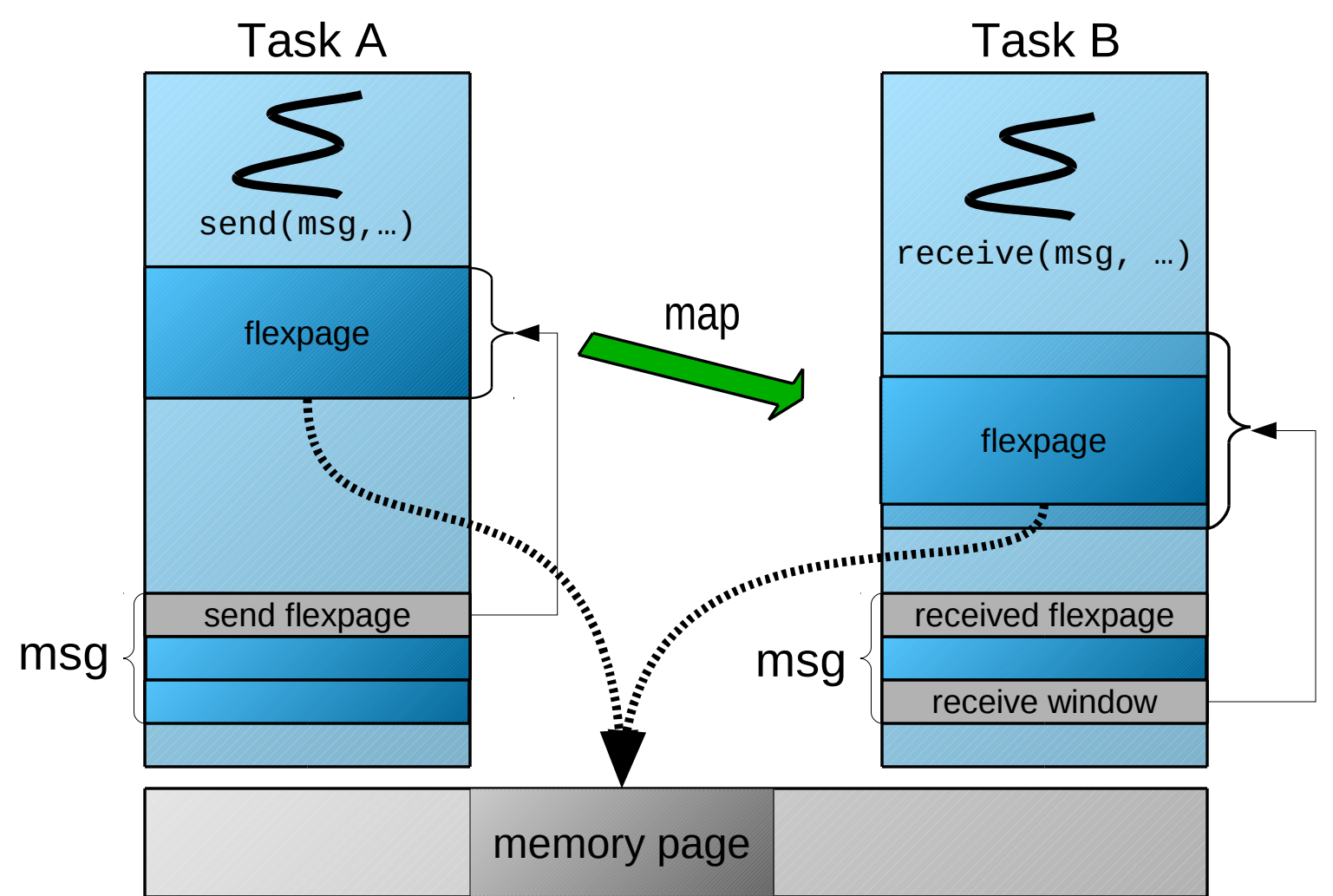




\section{Communication and Resource Control}

- Need to control who can send data to whom

- Security and isolation

- Access to resources

- Approaches

- IPC-redirection/introspection

- Central vs. Distributed policy and mechanism

- ACL-based vs. capability-based

Task B

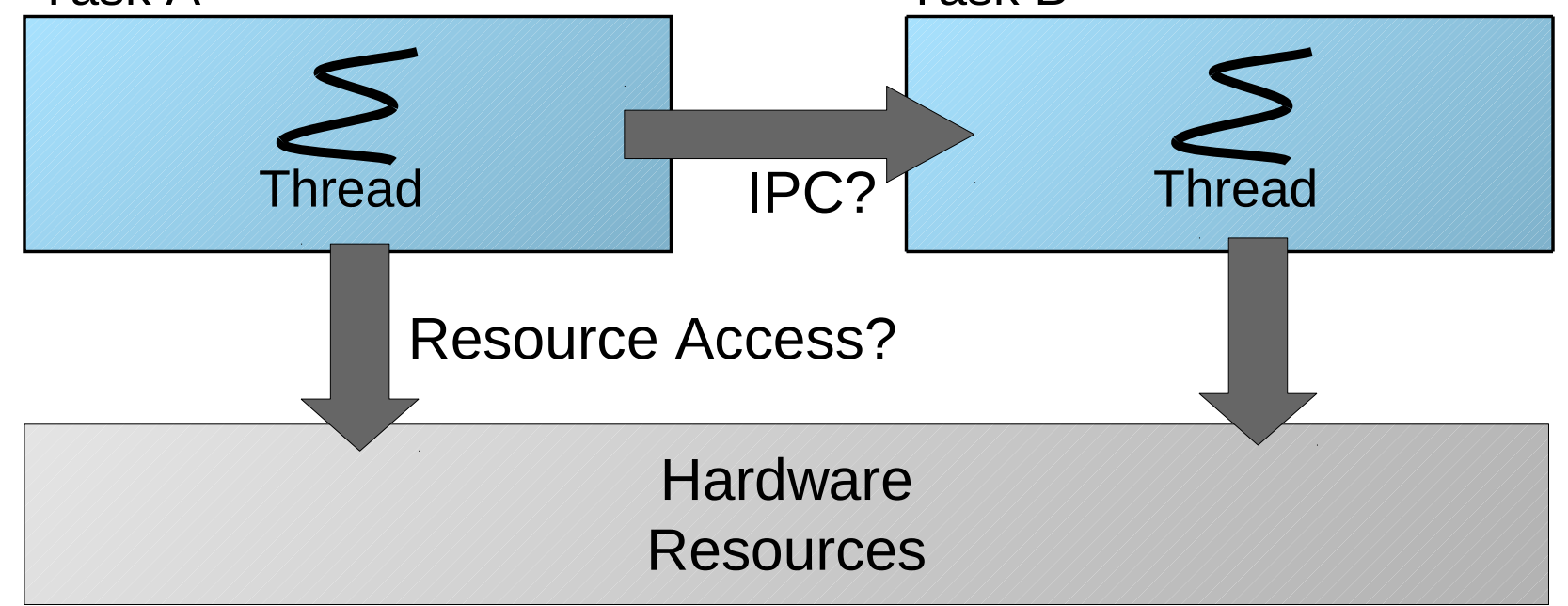




\section{Capabilities}

Task A

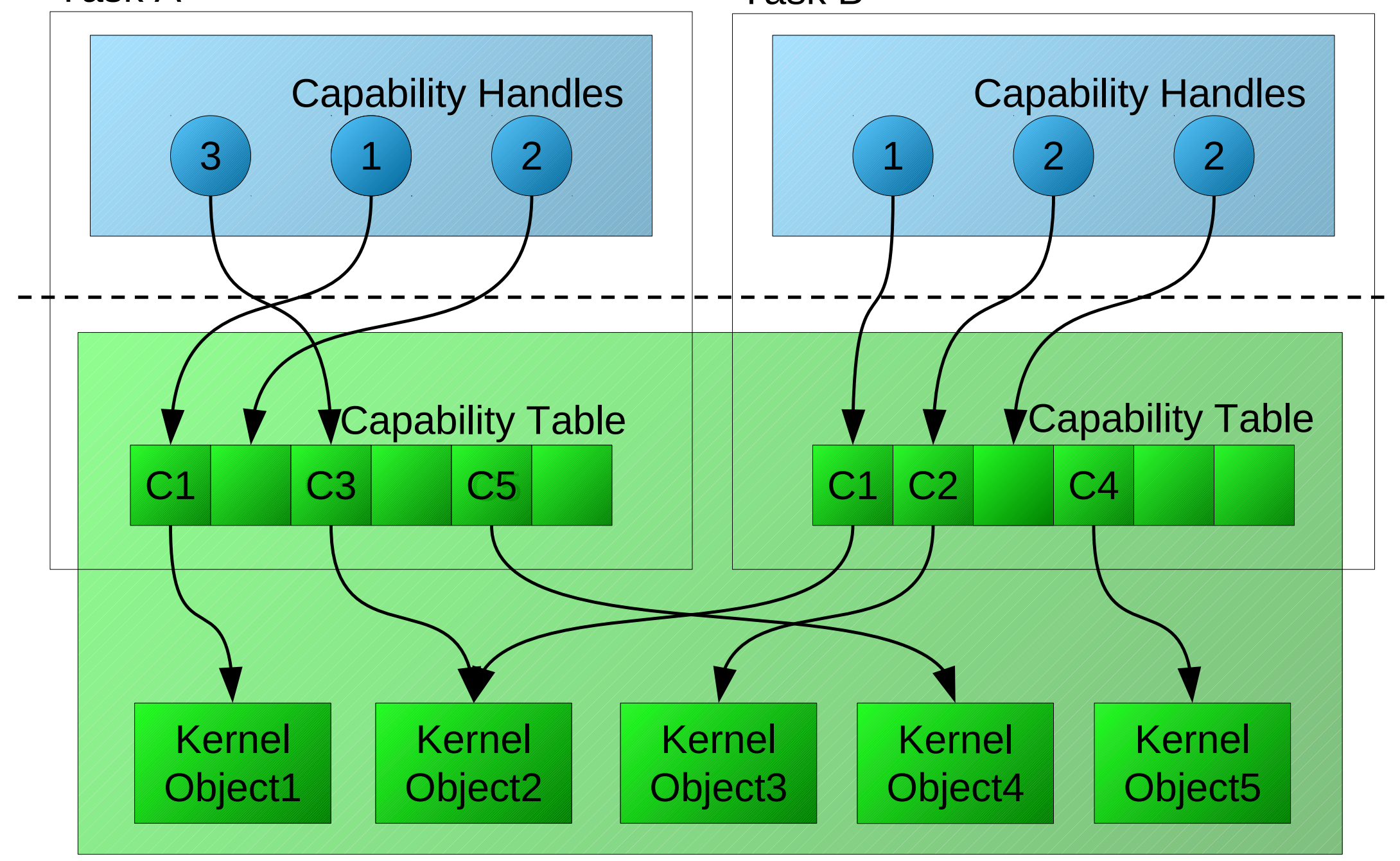

\section{Task B}

Capability Handles

$\checkmark$ Capability Table C1 $\mathrm{C} 2 \quad \mathrm{C} 4$ 


\section{Capabilities - Details}

- Kernel objects represent resources and communication channels

- Capability

- Reference to kernel object

- Associated with access rights

- Can be mapped from task to another task

- Capability table is task-local data structure inside the kernel

- Similar to page table

- Valid entries contain capabilities

- Capability handle is index number to reference entry into capability table

- Similar to file handle (in POSIX)

- Mapping capabilities establishes a new valid entry into the capability table 


\section{Page Faults and Pagers}

- Page Faults are mapped to IPC

- Pager is special thread that receives page faults

- Page fault IPC cannot trigger another page fault

- Kernel receives the flexpage from pager and inserts mapping into page table of application

- Other faults normally terminate threads

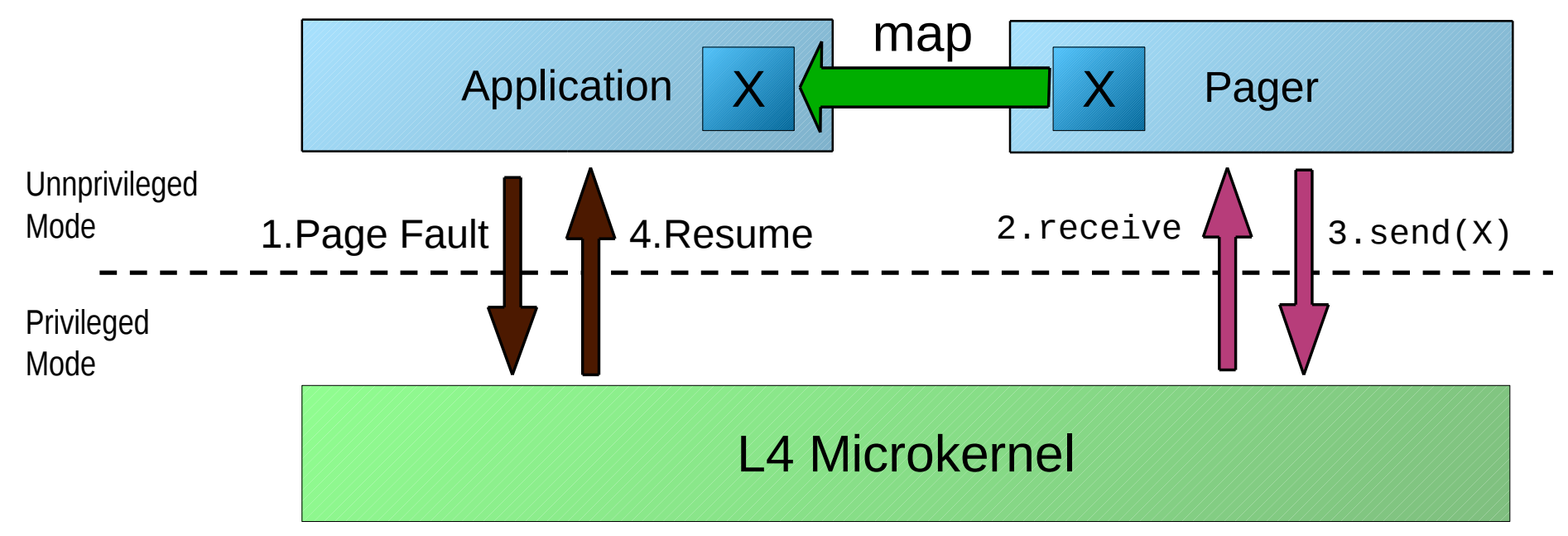




\section{Device Drivers}

- Hardware interrupts: mapped to IPC

- I/O memory \& I/O ports: mapped via flexpages

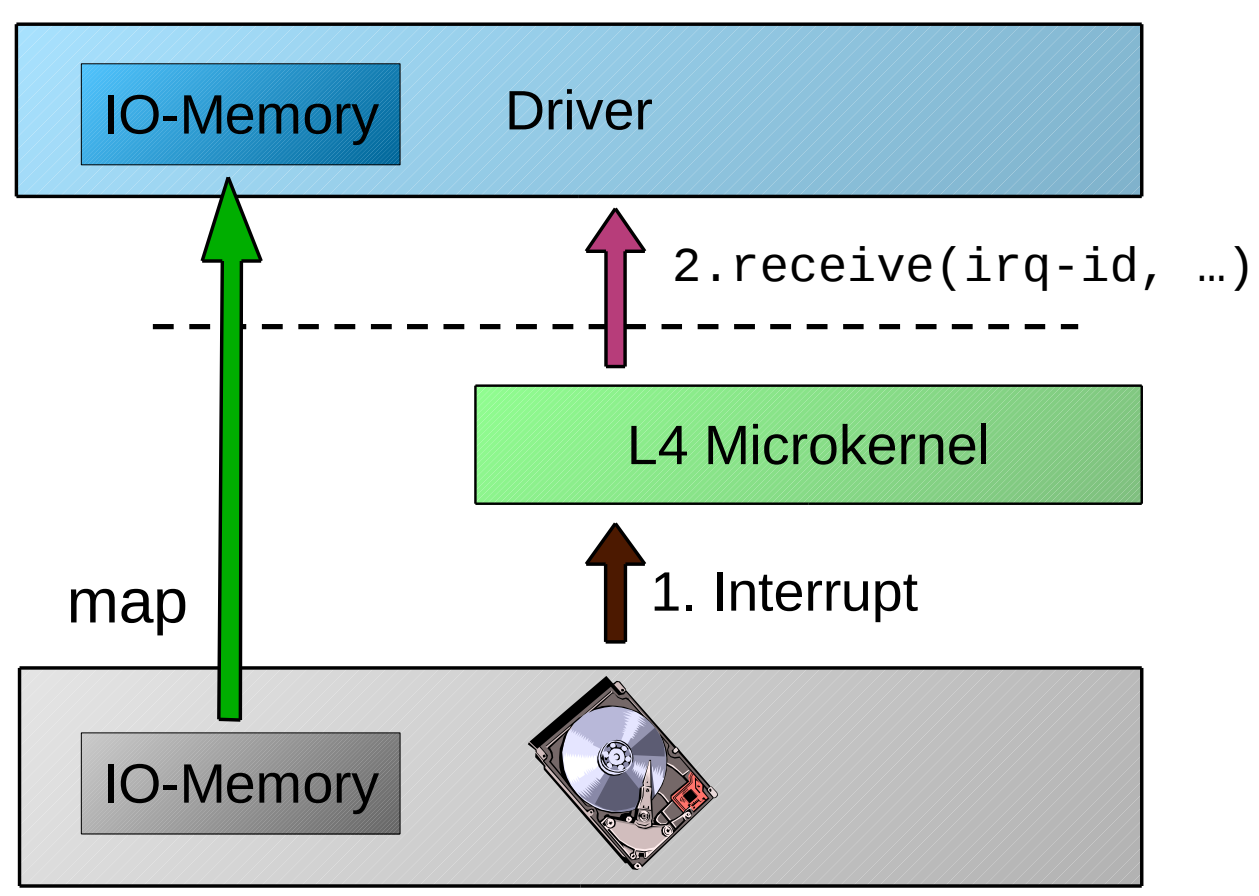




\section{L4 Applications - L4Linux}

- Paravirtualized Linux kernel and native Linux applications run as user-level L4 tasks

- System calls / page faults are mapped to L4 IPC
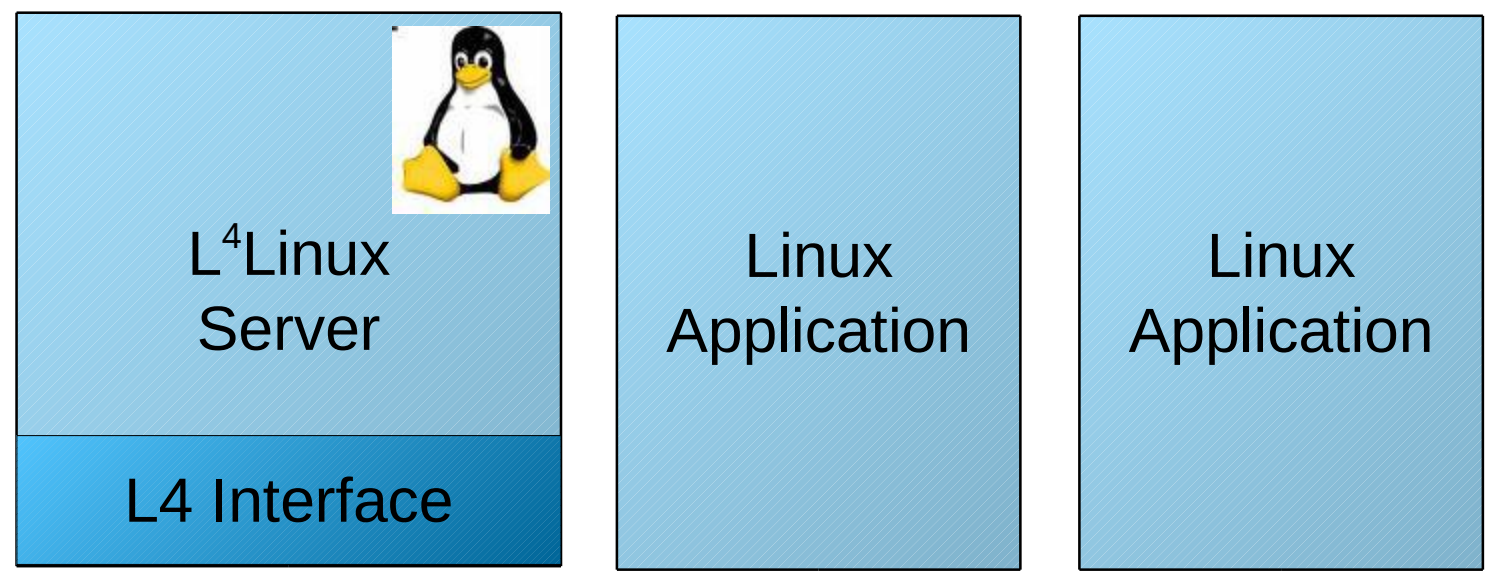

\section{System Services}

\section{L4 Microkernel}




\section{Lecture Outline}

- Introduction

- Address spaces, threads, thread switching

- Kernel entry and exit

- Thread synchronization

- IPC

- Address space management

- Scheduling

- Portability

- Platform optimizations

- Virtualization 